\title{
Complex Data-intensive Systems and Semantic Grid: Applications in Satellite Missions
}

\author{
M. Sánchez-Gestido, L. Blanco-Abruña \\ Deimos-Space \\ manuel.sanchez@deimos-space.com,luis.blanco@deimos-space.com \\ M. S. Pérez-Hernández, R. González-Cabero, A. Gómez-Pérez \\ Universidad Politécnica de Madrid \\ mperez@fi.upm.es,rgonza@fi.upm.es,asun@fi.upm.es \\ Ó. Corcho \\ University of Manchester \\ Oscar.Corcho@manchester.ac.uk
}

\begin{abstract}
The use of a Semantic Grid architecture can ease the deployment of complex applications, in which several organizations are involved and where resources of diverse nature (data and computing elements) are shared. This is the situation in the Space domain, with a strong demand of computational resources inscribed in an extensive and heterogeneous network of facilities and institutions. This paper presents the S-OGSA architecture, defined in the Ontogrid project, as applied into a scenario for the overall monitoring and data analysis in a Satellite Mission currently in nominal operations. Flexibility, scalability, interoperability and use of a common framework for data sharing are the main advantages of a Semantic Grid implementation in Complex and data-intensive systems.
\end{abstract}

\section{Introduction}

In the last years, complex applications have arisen in multiple domains. Many of these applications have been solved by means of traditional techniques. Although these solutions are feasible, they are also error prone and difficult to implement in practice.

In these scenarios, where a huge number of resources are shared and several organizations are involved, the use of grid computing can help to deploy flexible solutions [5]. If there are complex interactions between all the components and participants of the applications, it is possible to take advantage of the use of a common vocabulary and conventions, which provide semantics and interoperability. The Use Case for "Quality Analysis of Satellite Missions" in the Ontogrid project [7] is one example of this kind of scenarios, since the current operational system for the selected Satellite (EnviSat) has been developed through the use of traditional techniques (in what follows called QUARC, as opposed to the Semantic Grid implementation, SG-QUARC). This use case is intended to improve the possibilities for analysis of the product quality in satellite missions. On the other hand, Ontogrid has as main goal the explicit sharing and deployment of knowledge to be used for the development of innovative Grid infrastructures and for Grid applications, that is, the construction of reusable infrastructures for Semantic Grid applications.

This paper describes this use case and its adaptation to the semantic grid architecture developed in the Ontogrid project, S-OGSA [2]. It shows how a semantic grid architecture [3] can provide a large number of benefits when adapted to this kind of complex situations.

The rest of the paper is organized as follows. In Section 2, we describe the Envisat mission, specifying the overall structure of the whole satellite system. Section 3 describes the Use Case for the Analysis of Satellite products. Section 4 explains the set up of a Semantic Grid infrastructure for this Use Case, and section 5 shows the application of the Reference Semantic Grid architecture developed in Ontogrid (SOGSA). Section 6 lists a number of potential applications in other satellite systems. Finally, section 7 outlines the conclusions of this work.. 


\section{ENVISAT: An Earth Observation Satellite System}

Earth Observation can be defined as the science of getting data from our planet by placing in orbit a Hardware/Software element with several observation instruments, whose main goal is to obtain measurements from the Earth surface or the atmosphere. These scientific data is sent to Ground Stations and then processed in order to get meaningful information (i.e. images).

In a nutshell and putting aside other aspects, basic working of an Earth Observation Satellite System consists on a simple process that is repeated over time. The instruments on board the satellite act like cameras that can be programmed (very complex cameras nevertheless), taking "pictures" (images) of specific parts of the Earth at predefined times. Parameters for taking this pictures (like any camera would need to operate) and also parameters for the satellite general configuration, constitute the information included in the Mission Planning issued by the Mission Planning System (MPS), which is sent to the Flight Operation Segment (FOS), that in turn sends an equivalent information to a Ground Station and from there to the satellite. FOS converts the information in the Planning into the shape of MacroCommands (MCMD's). This means a translation from one planning format to another that is meaningful to the satellite. The Ground Station translates the MCMD information into a RadioFrequency link to communicate with the satellite antenna in the Service Module of the spacecraft.

A computer on board the satellite will store the list of MCMD's, each of them with a time tag that marks the execution time of that MCMD. Taking one "picture" would mean, for instance, the execution of a MCMD, which triggers a sequence of operations: Copy of those parameters for taking the picture, from the memory of the satellite computer to the instrument computer memory, and then the execution of the command that triggers the camera shot.

Each MCMD is a command (constituted by different pieces of information) that asks an instrument or any other part of the satellite to perform an action (e.g. load a table or trigger an operation).

The FOS also includes some additional MCMD's in order to get information from the Satellite internal status and configuration at a particular moment. Telemetry is the generic name for this information sent by the Satellite to the Ground Station for monitoring purposes.
"Pictures" from each of the instruments are stored onboard (in the satellite computer memory) as raw data and when the satellite over-flies the Ground station that data is sent to the Ground Station antenna (Data downlink). In that Ground Station a preliminary conversion from raw data to a so-called "Level 0 " product is performed (basically adding a identification label to each of the pictures). These "Level 0" products are sent to the Payload Data Segment facility that generates Level $1 \mathrm{~b}$ (with geo-location data) and then, after further processing, the final Level 2 products, which are made available to the final user community (scientist, environmental organizations, etc). Fig. 1 shows graphically the overall scenario. A more detailed explanation of the whole system can be found in [4].

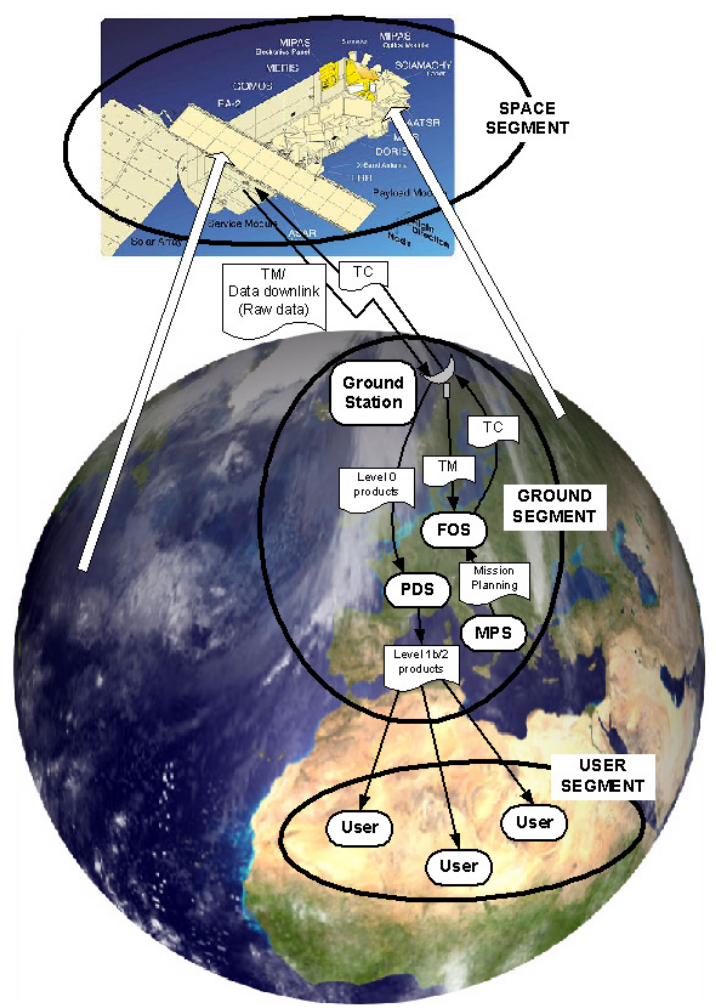

Fig. 1. General overview of an Earth Observation Satellite system (Envisat)

The Envisat satellite has an ambitious and innovative payload that ensure the continuity of the data measurements of the European Space Agency (ESA) ERS satellites. ENVISAT data supports Earth Science research and allows monitoring of the evolution of environmental and climatic changes. Furthermore, the data will facilitate the development of operational and commercial applications. The list of instruments for this mission is composed of 10 
instruments: RA-2, MWR, MIPAS, MERIS, GOMOS, ASAR, AATSR, DORIS, SCIAMACHY and LRR. Reference [10] contains extensive information describing the Envisat mission.

Data circulates within the system in the shape of files, with a well-defined structure. Mission Planning for the instruments and for the Satellite operations is issued regularly (background regional mission), nominally on a weekly basis, and can be later modified, before uplink to the satellite, upon arrival to FOS of requests for some instruments with High Rate modes (e.g. MERIS and ASAR). These requests have to be accommodated in the previous valid planning, that can be, therefore, modified several times before it is frozen. A catastrophic event (earth-quakes, volcano eruptions, hurricanes, ...) or a specific demand from the scientific community are examples of a last minute re-planning.

\section{Analysis of Satellite products: SG- QUARC Use Case definition}

QUARC is a system that checks off-line the overall data circulation process and in particular the quality of the instrument product files. This process needs as input the product files, the MCMD and the mission planning, provided to the system by other facilities. Apart from the product files in the PDS it needs to retrieve information from other parts of the system to crosscheck that the planning has been transformed properly into MCMD's, the instrument has performed successfully the measurements (taking pictures of the Earth), that these pictures have been stored onboard and transmitted as Raw Data to the Ground station and the processing from Raw Data to Level 0 and then to Level $1 \mathrm{~B}$ and Level 2 was correct

QUARC returns reports and plots, which helps in the production of a new planning. Therefore, the QUARC system is designed to assist when taking decisions in the situation where an instrument or the whole system begins to malfunction and to detect, in a semi-automated fashion that something incorrect has occurred in one part of the product generation or data circulation.

The QUARC data server obtains all the files needed from different facilities. The Data Ingestion Modules, one per file type, read the files and convert their contents into parameters that are meaningful to the QUARC data model (in the QUARC DataBase).

The operational QUARC system and all its elements (databases stored in computers, servers, ...) are attached to a single location (ESA-ESRIN, in Italy) that communicates with the archive containing all the products generated from the beginning of the mission (Envisat was launched in March 2002) and with all the other facilities.

\subsection{SG-QUARC: Use Case definition for "Instrument Product Unavailability"}

During routine operations, and except for regular calibrations, instruments on board the satellite are supposed to run continuously in Nominal mode. Some gaps may appear in the generated Level $1 \mathrm{~b} / 2$ product files, which is the final outcome from the instrument data. These product files are supposed to cover the time interval for which the instrument has been planned in Nominal mode and they may not be available in the system for a number of reasons (a different mode has been scheduled, a regular calibration is performed, etc).

When an instrument product file is not available, it is possible to infer the cause of this gap by tracing back the intermediate data and files associated to the sequence of events that should have generated that product. In order to do so the SG-QUARC system should be able, for instance, to generate and compare the timelines associated to the planning and the actual products (see Fig. 2). This is possible with the SGQUARC prototype that is currently under development, in a representative test environment using Envisat data, and with the additional difficulty of a completely distributed system (planning and product files).

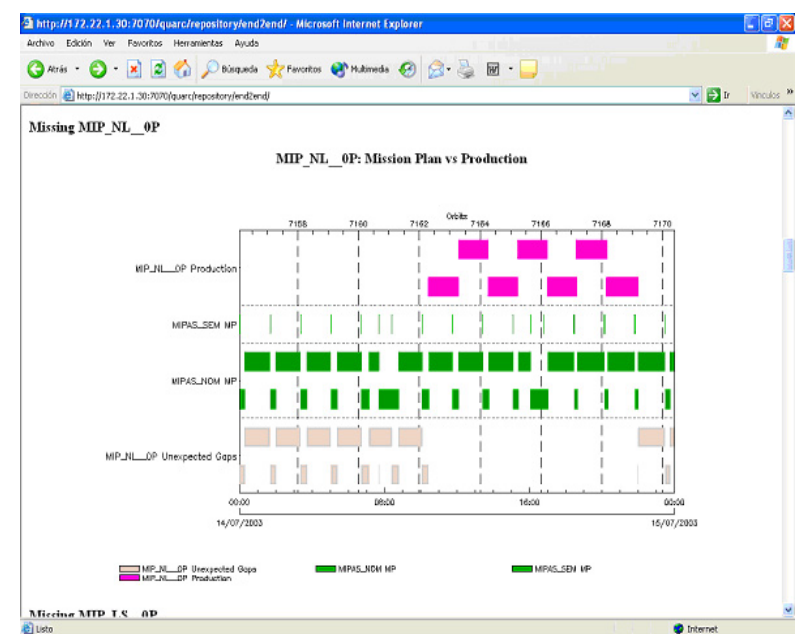

Fig. 2. Timeline for the MIPAS instrument (14Jul-2003)

\subsection{Requirements for the SG-QUARC use case}


Reference [4] poses a number of conditions to the SG-QUARC process that would represent stringent demands on a conventional approach. Some requirements for the implementation of the Use Case are worth mentioning:

- Definition of roles in the system, with permissions that allow or prevent retrieving specific pieces of information (with policies for limiting access) and/or the ability to perform tasks (this is materialized, in terms of implementation, by the possibility of forming Virtual Organizations).

- Remote operation of the system with access to data spread in different locations in a transparent manner to the user.

- Explicit description of links between diverse pieces of information in the system. These links may be used to define and perform queries to the system.

- Straight-forward adaptation from one Earth Observation (EO) mission to another EO satellite mission by specifying the specific aspects that differentiate both.

- Ability to name (using a generic reference scheme) and retrieve data contents from any file in the distributed system (or from any group of files defined with a specific criteria).

- Security policies to allow and deny access to specific parts of the system.

\section{A Semantic Grid infrastructure for the SG-QUARC process}

The QUARC system involves a complex process in which distributed data belonging to different facilities and organizations must be queried, processed and transferred. Suitable access control mechanisms have to be defined for these operations. Currently, the location of the resources and the processing of data are made in a wired way, according to filenames and content of these files in an "ad-hoc" format. The use of a grid framework provides a flexible way of locating required resources and the virtualisation of these resources by means of Grid Services.

Several resources used in this use case benefit from the generation and use of semantically-rich metadata, which is made up by means of an automated annotation process. This process is needed to link file resources with their actual content meaning (that is, to provide metadata about the data used in the system). Not only content should be annotated, but also provenance information, including temporal information (time stamp) about the data and the computational tasks involved in their generation. The explicit and formal relationships between operational parameters of the satellite instruments and the data obtained from them can also be represented in an ontology, and annotated from the actual planning of the instruments.

Fig. 3 presents a general overview of the SGQUARC process, with a centralized ontology describing the concepts and relationships between elements. Data (planning and product files) and associated metadata are completely distributed in a number of sub-facilities, breaking up the demands in terms of computational resources and disk storage. Within this distributed layout it is possible to perform queries on the annotated metadata, allowing the SGQUARC to retrieve information and build up more elaborated reports and plots which are eventually presented for the user.

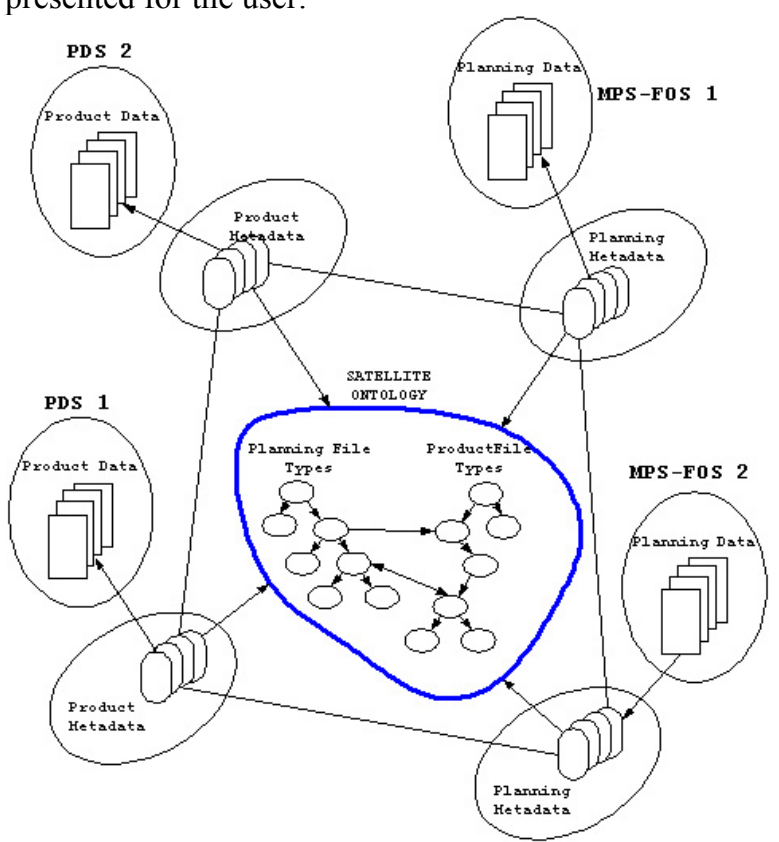

Fig. 3. Semantic Grid infrastructure for distributed file annotation and metadata queries

During nominal operation of a Satellite Mission, external facilities (external with respect to the QUARC system) are generating files on a continuous basis, with a frequency that varies from file type to file type. Due to the enormous amount of files circulating in the system and the huge size on the final products, the SGQUARC system could perform the annotation process on these files at different levels. On a regular basis, and for every arriving file, some basic information is annotated (e.g. time span for the data contents, instrument data, processing facility, ...). Later on, and only in association with a query performed by the user, a more complete annotation can be performed for the 
contents of the particular files relevant to the output of the query. This on-demand annotation process reduces the amount of metadata stored in the system to the actual needs of the user.

Regarding security, the involvement of several organizations implies the establishment of different access policies and the definition of Virtual Organizations (VO). The role of each specific actor within an organization also defines its privileges as a member of the VO. A VO prototype developed as part of the OntoGrid project has already proved that VO's can be created and dismantled.

\section{S-OGSA in the SG-QUARC process}

S-OGSA [13] is the reference Semantic Grid architecture developed in Ontogrid. It is described in the next section. After this description we describe the application of this architecture in the context of the SG-QUARC process.

\subsection{S-OGSA}

The basis of S-OGSA is an information model of semantic resources, which extends the OGSA model [14]. Besides, S-OGSA anticipates that a set of capabilities will be required from Grid middleware in order to address the new services with varying degrees of semantic capabilities. For this, S-OGSA includes two service categories called Semantic Provisioning Services and Semantically Aware Grid Services.

5.1.1. S-OGSA Information Model. The S-OGSA model identifies two groups of entities:

- Grid Entities are anything that carries an identity on the Grid, including resources and services [15].

- Knowledge Entities are special types of Grid Entities that represent or could operate with some form of knowledge. Examples of Knowledge Entities are ontologies, rules, knowledge bases or even free text descriptions that encapsulate knowledge that can be shared.

A special type of Knowledge Entities are the socalled Semantic Bindings, which are entities that represent the association of a Grid Entity with one or more Knowledge Entities (that is, they represent semantic metadata of a Grid Entity). Existence of such an association transforms the subject Grid entity into a Semantic Grid Entity. Semantic Bindings are first class citizens as they are modelled as Grid resources with an identity and manageability features as well as their own metadata.
Semantic Grid Entities are those Grid Entities that are either the subject of a semantic binding, are themselves a semantic binding, or a Knowledge Entity. Grid Entities can be associated with zero, one or multiple knowledge entities of different forms and capabilities, simultaneously, and can acquire and discard associations with knowledge entities through their lifetime.

5.1.2. Semantic Provisioning Services. These are Grid Services that provision semantic entities. Two major classes of services are identified:

- Knowledge provisioning services, which can produce (and in some cases store and manage) Knowledge Entities. Examples of these services are ontology services and reasoning services.

- Semantic Binding provisioning services, which can produce (and in some cases store and manage) Semantic Bindings. Examples of these services are semantic binding index services, for accessing and storing metadata associating Grid Entities with Knowledge Entities; and annotation services for generating metadata from different types of information sources, like databases, files or provenance logs.

5.1.3. Semantically Aware Grid Services. These special type of Grid Services are able to exploit semantic technologies to consume Semantic Bindings in order deliver their functionality. Their role is complementary to the role of Semantic Provisioning Services since they consume the semantic entities held by Knowledge provisioning services and Semantic Binding provisioning services, and use their services.

\subsection{S-OGSA in the SG-QUARC process}

In this section we analyze, from an architectural point of view, which are the most important elements of S-OGSA that are applied in the improved QUARC process. We start first with the information model of this system, where we can identify the following types of entities:

- Grid Entities: planning systems, planning files, satellite instruments, product files and product processing facilities. These entities would be considered similarly in any non-semantic implementation of the process.

- Knowledge Entities. A set of ontologies in the satellite mission domain. These set includes a range of different types of ontologies with different levels of usability and reusability, from core ontologies related to time, to more application-oriented ontologies used 
to describe planning systems, macrocommands, satellite instruments, etc.

- Semantic Bindings are generated through an annotation process of the different types of files that are generated in the QUARC process. They are attached to these files (which are Grid Entities themselves, as aforementioned) as described in the SOGSA information model section, making these Grid Entities become Semantic Grid Entities.

The Semantic Provisioning Services used in this system are:

- A set of ontology services that store the set of ontologies about the domain and the application. These ontology services are implemented using WS-DAIOnt [12][36], also developed in the context of the OntoGrid project.

- An annotation service that generates Semantic Bindings from planning and product files. This annotation service is implemented using the Knowledge Parser tool.

- A storage service for the Semantic Bindings that are generated, so that they can be accessed at any time during their lifetime using a query language. This service is implemented using the Atlas system [11][37].

Finally, there is only one Semantically Aware Grid Service, which is the SG-QUARC itself, whose functionalities were described in sections 3 and 4 . This service makes use of all the aforementioned elements in order to deliver its enhanced functionality.

\section{Semantic Grid benefits to Satellite Missions}

One of the main advantages of a Semantic Grid approach in Satellite Missions is that, once the conceptualisation of generic elements of the system is done, most of the features are reusable from one Satellite Mission to another.

\subsection{Earth Observation Satellite Missions}

Although the explanations in section 2 might suggest the opposite, an Earth Observation Satellite mission is a very complex process, with a large number of diverse conceptual elements. Some characteristics are a common-place in these Satellite Missions:

- It demands dealing and processing enormous amounts of scientific data with very different characteristics. According to [9], "Only 3 months after launch, Envisat data dissemination system for distributing satellite imagery $[\ldots]$ reached the terabyte mark of data transferred over the satellite channel".

- There are thousands of different parameters with scientific meaning involved in the computations and it is necessary to extract a limited amount of them to be interpreted by the end user.

- It requires highly specialized engineers alongside with system engineers, each of them having very different views on the system.

- System development means dealing with a large number of companies and with several levels of subcontracting.

- Complicated problems may arise from instruments outages. Some of them require particular offline analysis by gathering a specific technical team from different areas in the mission.

- A single satellite carries, in general, several instruments with specific needs that, during operations, may be conflicting in terms of power, field of view obstruction or data downlink. A proper planning ahead should solve these questions in advance.

- Satellite data downlink can involve a Satellite Communication network and/or several Ground Stations. At the same time, Data handling and product processing is performed by several facilities (Fig. 4). Both conditions affect to the amount of data circulating in the system.

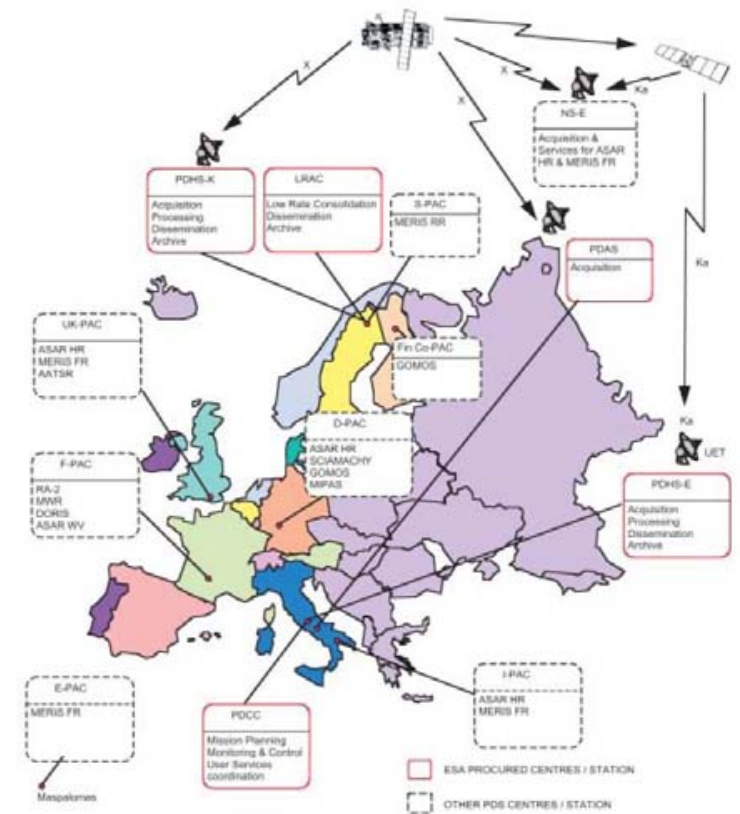

Fig. 4. Locations and primary functions of the various Payload Data Segment (PDS) centers and Stations in the Envisat mission 
Earth observation is a long-term policy where satellite missions are defined to continue and improve accuracy and coverage of measurements in terms of world coverage but also in terms of continuity of data (for instance to oversee trends during a period of several years or decades in ozone concentration over the Antarctica continent). It also means combine data (images) obtained from satellites developed by other Space Organizations (NASA, NASDA, National Space Agencies in Europe, etc.)

As a complementary objective, Earth Observation missions aim at providing quick reaction in the event of natural disasters. It requires an appropriate infrastructure for a coordinated and accurate response in those circumstances [9].

\subsection{Semantic Grid benefits for Earth Observation Satellite Missions}

As a first step in order to automate processes and tasks in complex systems it is necessary to share, between the different elements in the system. Semantics provides a common understanding of abstract concepts, actual objects and actions that can be performed on them. At the same time, Semantics associates this description to conceptualisations in standard Knowledge Description formats that allow using generic and powerful software tools to query any part of the system for relevant information in a seamless way to the user.

Ontologies make feasible the partial annotation and identification (by several criteria) of relevant pieces of information within the files, instead of keeping an actual copy of all the contents of those files. As long as the amount of data produced by every Satellite Mission is enormous, it is a tremendous advantage, on a routine basis, to just keep track of the relevant information in order to be able to access, at a later step, the actual information needed by the users, and at that time, request the full content of the product from the archive. Access on demand to Satellite system information requires retrieving files from several areas in the system (planning, MCMD generation, etc) that can be spread in several physical locations. This is in accordance with the current evolution of the Processing Data Centers for Satellite products with distributed computations and selective on demand processing.

Annotation and meta-data information, added on a routine basis, are useful to orientate the user request to search for additional information. Not only the annotation of data, but also provenance annotation (when it was produced, by whom, which files were used for that, etc). For instance, that is useful to identify the cause of an instrument problem by tracing back the events that led to the failure.

The Semantic Grid implementation can be done in an incremental way, considering some well-defined parts of the system temporarily as not changed and then, step by step, broadening the scope of the Semantic Grid implementation. This is made possible by:

- Encapsulating computational resources.

- Translating internal data formats to agreed interfaces (this also allows to keep working internally with formats that are convenient for specific purposes).

Semantic Grid goes one step further and may be viewed as a layer on top of Grid Computing and Common Data Description schemes, putting everything together so enhanced functionalities arising from both approaches can be implemented in a common software frame.

Semantics make possible the interaction of heterogeneous elements in the system. This is particularly useful when setting up working groups that are going to work together for a limited period of time, sharing resources and communication links. It is supported by Virtual Organization forming.

The additional implementation effort in formalizing and making explicit the domain knowledge is compensated by far with the benefits in terms of expandability, interoperability (standardized approach), scalability and maintainability (use of generic and reusable elements).

\subsection{Extensions to other Satellite Missions}

New ways of cooperating and sharing resources in Earth Observation Satellite missions are under way. In particular, the objective of the Global Monitoring Environment and Security (GMES) initiative is to provide products from several Earth Observation missions to many interdisciplinary institutions, science and business-oriented users [16],[17].

There is an international on-going standardization effort within the Space community, lead by the Consultative Committee for Space Data Systems (CCSDS) in order to enhance interoperability and cross-support between Space Agencies. These initiatives aim at the definition of generic concepts and common representation paradigms and software architectures that allow sharing and re-using file-based information managed by different systems [18]-[20].

It is worth noting that most of the characteristics depicted for Satellite systems can be identified in other scientific and engineering domains, e.g. air traffic 
control and astronomy where Grid technologies are being applied [21],[22].

In relation to other Satellite Missions with complementary uses, Meteorological and Geographical Information Systems (GIS), Global Navigation Satellite Systems (GNSS, like the widely used GPS) need also to combine huge amounts of scientific data coming from different sources [23],[24].

Emerging Grid Technologies concepts [25], Digital libraries [8], [27], [28], [29], standard definitions for data Format and structure [30]-[32], are gradually incorporated into all those complex systems. The Semantic Grid aims at unifying all those approaches and technologies so they can interoperate and build on a common effort.

\section{Conclusions}

This paper has addressed the use of a semantic grid architecture in a complex environment, such as a satellite mission system. The benefits of the deployment of such discipline have been also shown, from two different points of view: the generic application of concepts from semantic grid and the actual application of a reference semantic grid architecture (S-OGSA) over a concrete use case. The lessons learn from this task can help us to extend this work to other different satellite missions.

\section{Acknowledgment}

The authors would like to thank the other members of the OntoGrid consortium for many thoughtful discussions. The authors are also very grateful to the European Space Agency (ESA) representatives Olivier Colin (ESA-ESRIN) and Pierre Viau (ESA-ESTEC) for providing access to the actual products and auxiliary tools from the Envisat mission.

\section{References}

[1] Arpírez J.C., Corcho O., Fernández-López M., and Gómez-Pérez A 2003.: WebODE in a nutshell. AI Magazine.

[2] Ioannis Kotsiopoulos et al. Specification of a Semantic Grid Architecture. September 2005. Ontogrid Deliverable D2.1. Available: http://www.ontogrid.net

[3] D. De Roure, N. R. Jennings, and N. R. Shadbolt, "Research Agenda for the Semantic Grid: A Future e-Science Infrastructure," National e-Science Centre, Edinburgh, UK UKeS-2002-02, December 2001.

[4] M. Sánchez Gestido. "OntoGrid Business Case and User Requirements Analysis and Test Set Definition For Quality Analysis of Satellite Missions". October 2005. OntoGrid Deliverable D8.1. Available: http://www.ontogrid.net

[5] I. Foster and C. Kesselman, "The Grid: Blueprint for a New Computing Infrastructure”. Morgan Kaufmann, 1999.
[6] ESA bulletin 106 "Envisat Special issue". Available: http://www.esa.int/esapub/pi/bulletinPI.htm

[7] Ontogrid Project. http://www.ontogrid.net.

[8] "Earth Observation (EO) Archives in Virtual Digital Libraries and Grid Infrastructures: an European view". The ERPANET/CODATA International Workshop on the selection, Appraisal, and Retention of Scientific Data. Lisbon, 15-17 December 2003. Available at: http://www.erpanet.org/events/2003/ lisbon/presentation Luigi Fusco

[9] "Envisat breaks the terabyte barrier", ESA news, 25 June 2002. Available:

http://www.esa.int/esaEO/ESALS5OED2D index 0.html

[10] ESA bulletin number 106, "EnviSat special issue". Available: http://www.esa.int/esapub/pi/bulletinPI.htm

[11] Atlas deliverable (OntoGrid). http://www.ontogrid.net

[12] WS-DAIOnt (OntoGrid deliverable). http://www.ontogrid.net

[13] O. Corcho, P. Alper, I. Kotsiopoulos, P. Missier, S. Bechhofer, D. Kuo, and C. Goble. An overview of s-ogsa: a reference semantic grid architecture. Journal of Web Semantics, 4, 2006.

[14] I. Foster, H. Kishimoto, A. Savya, D. Berry, A Djaoui, A. Grimshaw, B. Horn, F. Maciel, F. Siebenlist, R. Subramaniam, J. Treadwell, and J. V. Reich. The open grid services architecture, version 1.0. Technical report, Open Grid Services Architecture WG, Global Grid Forum, 2005. A new version has just been released and is under public comment.

[15] J. Treadwell. Open grid services architecture glossary of terms Technical report, Open Grid Services Architecture WG, Global Grid Forum, 2005. Available at: http://forge.gridforum.org/projects/ogsa-wg. A new version has just been released and is under public comment.

[16] "GMES: Visions and targets 2006", 3rd GMES Forum, May 2003, available at: http://www.gmes.info/library

[17] "ESA Earthwatch Programme: GMES Service Element", available at: http://www.gmes.info/library/

[18] "Reference Model for an Open Archival Information System (OAIS). Blue Book". Issue 1. January 2002. Available at: http:// http://public.ccsds.org/publications

[19] "Statement of Work for Generic Exchange Format and Interface Standardisation for Ground Segment", available at: http://emits.esa.int/emits

[20] M. Bertelsmeier, G. Buscemi, "New Communication Solutions for ESA Ground Stations", ESA Bulletin number 125, Feb 2006

[21] International Virtual Observatory Allaince: http://www.ivoa.net/

[22] AstroGrid: http://www.astrogrid.org/

[23] Stefano Nativi, "ISO TC2111: Geographic Information - Imagery, gridded and coverage data framework"

[24] S. Nativi "THREDDS vs OpenGIS: recent notes"

[25] L. Fusco et al. "Open GRID services for Envisat and EO applications". Available: http://goncalveswww.eogeo.org/Workshops/EOGEO2004/ eogeo2004-grid/eogeo-2004-goncalves

[26] Internet Data Distribution: http://www.unidata.ucar.edu/software/idd

[27] Earth Observation and Geospatial Information and Communication Technologies (EOGEO), http://www.eogeo.org

[28] The Earth Observation System (EOS), http://eospso.gsfc.nasa.gov

[29] The Earth Observatory, http://earthobservatory.nasa.gov

[30] Unidata: http://unidata.ucar.edu

[31] Geospatial Data Abstraction Library (GDAL), http://www.remotesensing.org/gdal

[32] Binex: Binary Exchange Format, http://binex.unavco.org

[33] Gómez-Pérez A., Estebán-Gutierrez M. and Muñoz-García, "Ontology Access in Grids with WS-DAIOnt and the RDF(S) Realization", $3^{\text {rd }}$ Semantic Grid Workshop, Feb. 2006.

[34] Miliaraki I., Koubarakis M. and Kaoudi Z. "Semantic Grid Service Discovery using DHTs", $1^{\text {st }}$ CoreGrid WP2 Workshop on Knowledge and Data Management, September 2005. 